\title{
Femoral Neck Stress Injury with Negative Bone Scan
}

Dennis Y. Wen, MD, Tim Propeck, MD, and Amolak Singh, MD

The cause of hip and groin pain can be difficult to diagnose because the symptoms are often nonspecific and can be attributed to a wide range of possible conditions. Stress fracture of the femoral neck must be kept in mind when evaluating hip and groin pain in active patients. The consequences of missing such a diagnosis are potentially devastating because of the problematic nature of these injuries. Diagnostic imaging is often used to evaluate possible femoral neck stress fractures. Plain radiographic findings often do not appear until late in the clinical course or sometimes not at all. A bone scan or magnetic resonance imaging (MRI) is therefore advocated as the test of choice for diagnosing femoral neck stress fractures. We report a case of stress injury to the femoral neck. There were negative findings on a bone scan, and the injury was ultimately diagnosed by magnetic resonance imaging (MRI).

\section{Case Report}

A 44-year-old healthy male attorney complained of an approximately 1-month history of intermittent left groin and lateral hip discomfort. He was in the military reserves and was quite active, running 2 to 4 miles several days a week and using an exercise bicycle and elliptical exerciser. Without any antecedent trauma, he began noticing the insidious onset of pain during the landing phase while running. He had stopped running about 2 weeks earlier but continued to cross-train with the exercise bicycle and elliptical machine, neither of which produced any discomfort. Walking was painless, but descending stairs caused mild pain. He reported no radicular symptoms. He was otherwise healthy and took no medications. He had had an arthroscopic partial

Submitted, revised, 20 November 2002.

From the Department of Family and Community Medicine (DYW), and the Department of Radiology (TP, AS), University of Missouri-Columbia. Address reprint requests to Dennis Y. Wen, MD, Department of Family and Community Medicine, University of Missouri-Columbia, M228 Medical Sciences Building, Columbia, MO 65212. medial meniscectomy of his left knee 7 months earlier and was asymptomatic.

When examined, he was a well-nourished male. Sciatic nerve tension signs for radiculopathy, including straight-leg raise, were negative. There was no tenderness in the groin, the greater trochanter, the anterior superior iliac spine, or the anterior inferior iliac spine. Passive range of motion was normal, but internal rotation while in flexion reproduced his pain. External rotation while in flexion was mildly uncomfortable. Resisted knee extension with the hip in extension produced pain, as did resisted hip extension. Resisted hip abduction and adduction were normal. Single-legged hopping with the left leg reproduced his symptoms deep in his groin. Plain films were unremarkable.

A hip flexor muscle strain was considered, as was a femoral neck stress fracture. He was advised not to run, but crutches were not instituted. An MRI could not be scheduled for 4 weeks, so a bone scan was ordered in the meantime.

Ten days later he had normal findings on a technetium-99m triple-phase bone scan (Figure 1). Single photon emission computed tomography (SPECT) images (Figure 2), however, showed a slight increase in uptake in both femoral necks near the lesser trochanters, with the left greater than right. By this time, he had symptomatically improved. Resisted hip flexion or knee extension was no longer painful on examination. Despite the nonspecific SPECT findings, it was believed that the normal bone scan findings essentially ruled out a stress fracture, and he was allowed gradually to resume running.

The groin pain recurred when he resumed running, and he therefore kept his appointment for the MRI, which was performed about 4 weeks after his initial visit. The MRI showed an increased signal on T-2 weighted and fat-suppression sequences in the medial aspect of the femoral neck (Figure 3), consistent with bone marrow edema. No discrete fracture line could be observed. Because of the clinical symptoms and signs, the MRI evidence of 


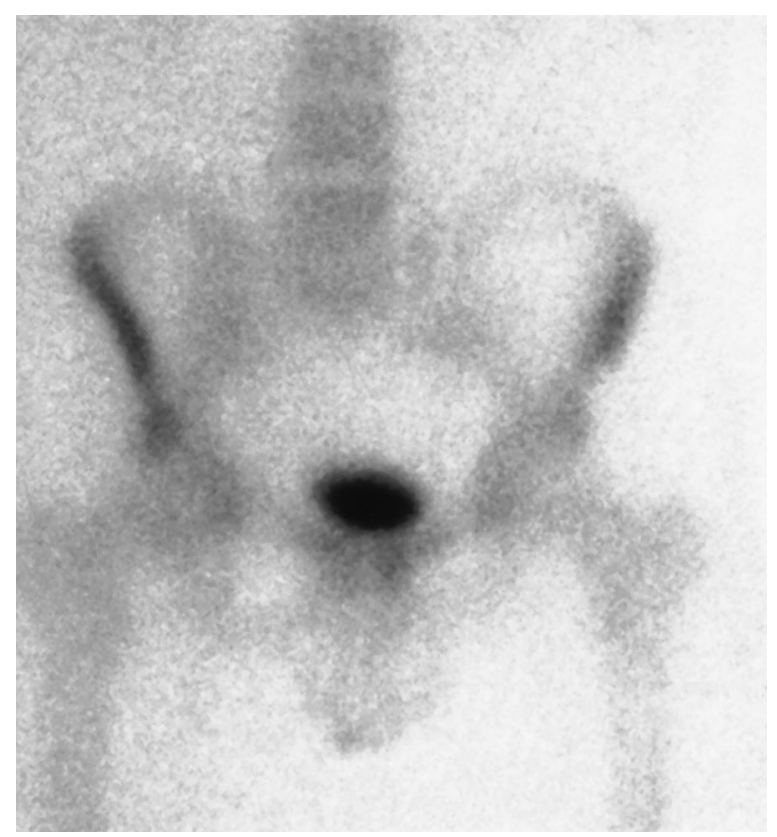

Figure 1. Planar technetium-99m bone scan (delayed image) of anterior view of the hips, about 5 weeks after symptom onset, shows no abnormalities.

bone stress injury, and the equivocal SPECT findings, the patient was advised to discontinue running for 3 months. He was allowed to walk without crutches and to exercise with an exercise cycle.

Three months later (4 months after the initial examination) he was asymptomatic, and findings during a physical examination were normal. Repeated plain films were negative for a stress fracture. Repeated MRI findings were now normal (Figure 4). The patient was allowed to resume running. He has since returned to his previous level of running and remains asymptomatic after 5 months.

\section{Discussion}

The incidence of stress fractures varies with the population studied, but it is common in runners, with estimates ranging from $9 \%$ to $31 \%$ of track and field athletes. ${ }^{1}$ Femoral neck stress fractures comprise $5 \%$ to $10 \%$ of all stress fractures. ${ }^{2} \mathrm{Al}-$ though uncommon, stress fractures of the femoral neck have a high complication rate, especially if the diagnosis is missed. Clinically the symptoms are generally poorly localized groin or anterior thigh pain of insidious onset and made worse by weight bearing. Physical examination findings are often nonspecific and include pain with passive internal rotation of the hip and reproduction of the pain

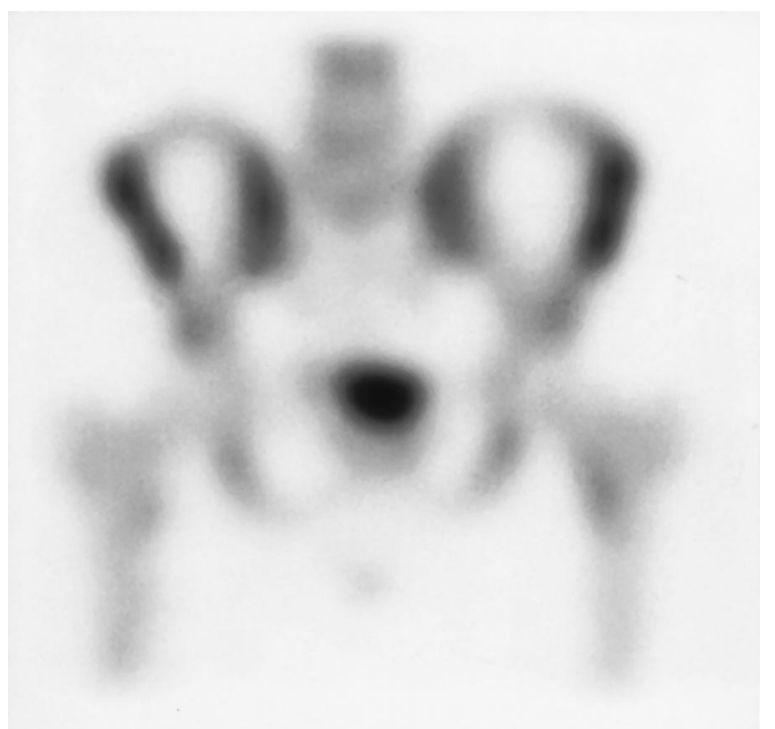

Anterior

Figure 2. Single photon emission computed tomographic (SPECT) image at the time of the bone scan shows slight increased uptake in the areas of both femoral necks near the lesser trochanters, left more than right. The left side was symptomatic.

with single-legged hopping. Our patient had typical clinical findings consistent with the possibility of a femoral neck stress fracture.

Radionucleotide bone scans have had a long history of reliability and extremely high sensitivity in the detection of bone stress injuries. ${ }^{3-5} \mathrm{~A}$ triplephase bone scan consists of a blood flow phase, a blood pool phase, and delayed bone images. Bone scans show uptake in areas of bone with increased osteoblastic activity, where the body is laying down new bone in an attempt to heal the stress fracture. In an acute stress fracture, abnormalities can generally be observed on all three phases of the scan. Bone scans can also show areas of increased uptake that are asymptomatic; these areas have been termed stress reactions. ${ }^{6}$ The importance of stress reactions is unclear, but it is postulated that some might become symptomatic (becoming actual stress fractures) if provocative activity is continued. The use of MRI has also been studied for the detection of stress fractures, ${ }^{7}$ and several studies have compared MRI with bone scanning for stress fracture detection and grading. ${ }^{5,8-10}$ Most investigators have concluded that MRI is as sensitive as bone scanning (almost 100\% sensitive) and much more specific. $^{9,10}$ 


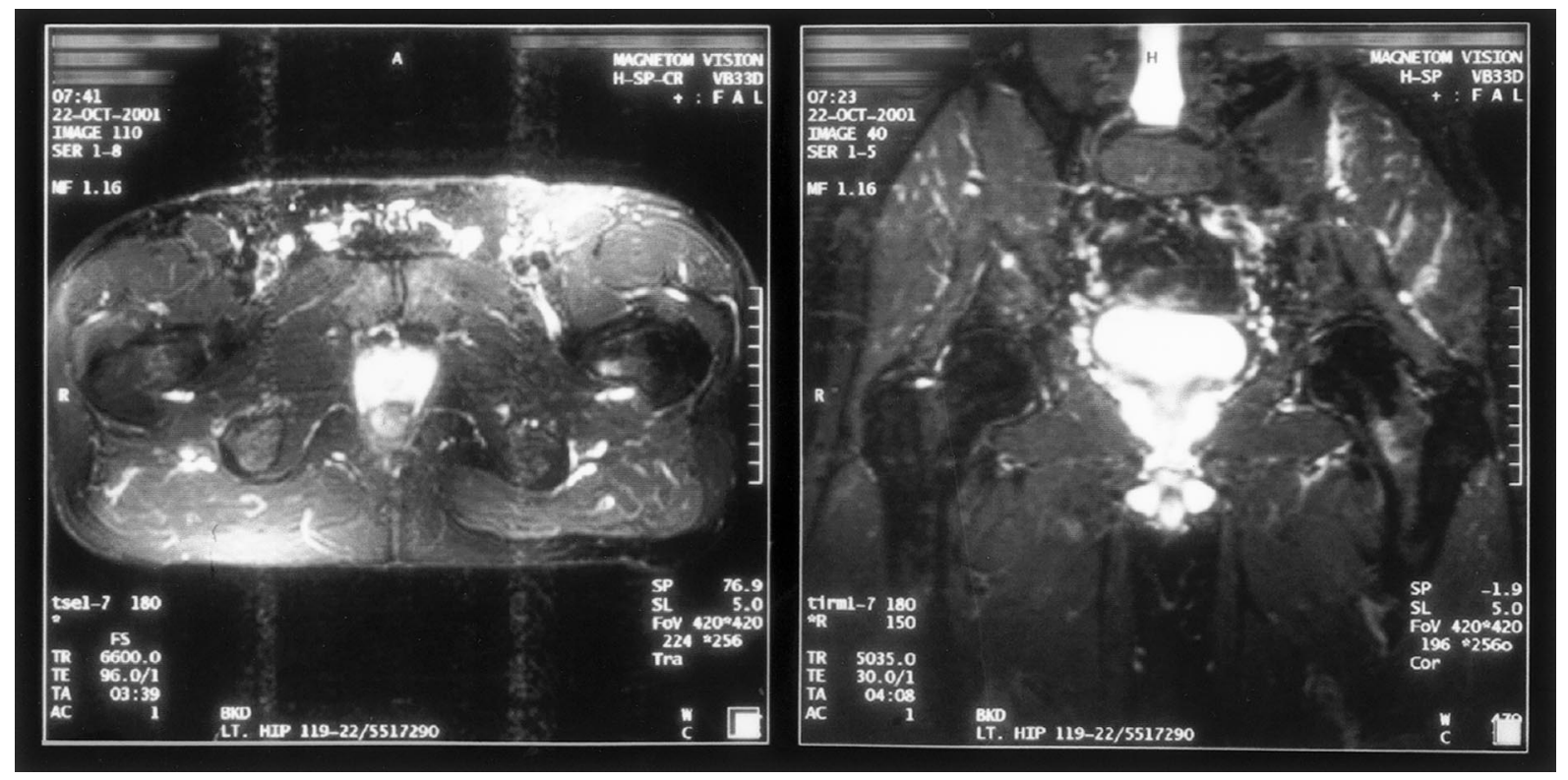

Figure 3. Magnetic resonance imaging of the left hip about 8 weeks after symptom onset, with STIR sequences; axial (left) and coronal (right) views show increased signal in the medial aspect of the femoral neck consistent with bone marrow edema.

Our patient had MRI evidence of femoral neck bone stress injury manifested as bone marrow edema on the T-2 weighted and fat-suppressed images. It is not clear that this finding truly represented a stress fracture, because no fracture line could actually be seen. The absence of a fracture

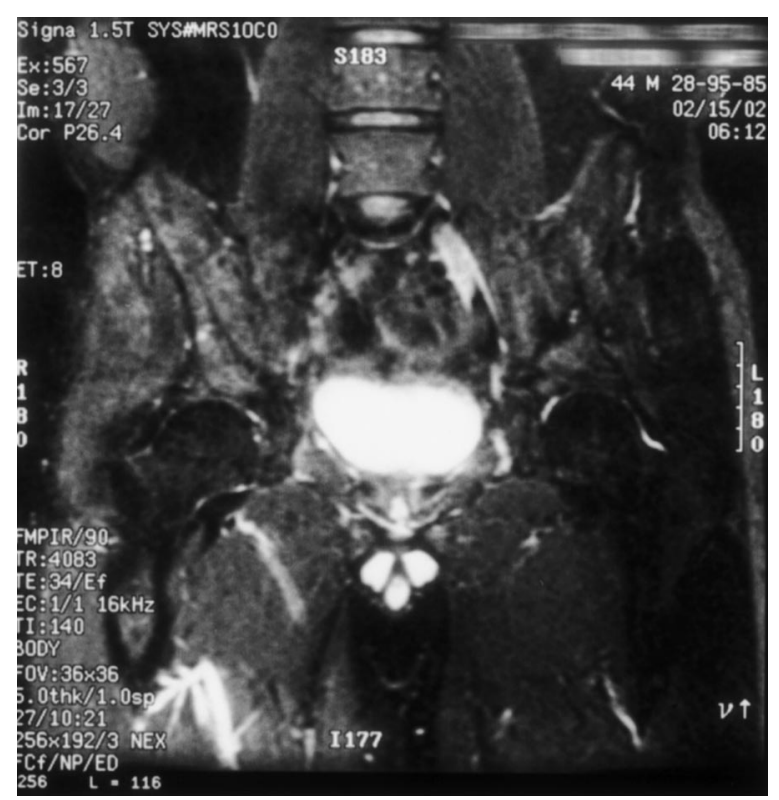

Figure 4. Follow-up magnetic resonance imaging 5 months after symptom onset shows no abnormalities in the femoral neck. Patient was asymptomatic. line does not, however, rule out a stress fracture. ${ }^{8}$ It is possible that our patient suffered a milder bone stress injury than an actual fracture, which would explain the lack of findings on bone scan, as well as the rather mild bone edema noted on the MRI. What this condition represents histologically or pathologically, which was not available in this case, would be difficult to explain.

The mild uptake found on the SPECT images also suggests some type of bone injury, whether a stress fracture or otherwise. The planar bone scan images were falsely negative. The SPECT images showed a femoral lesion that was thought to represent a stress injury on the MRI, because the plain film radiographs and history were negative for other conditions, such as traumatic fracture or bone neoplasm. SPECT imaging involves computergenerated tomographic images of planar bone scans, thus increasing the contrast resolution and improving detection and localization of small abnormalities. The use of SPECT scanning has been more extensively evaluated for detection of stress fractures of the pars interarticularis of the spine, ${ }^{11,12}$ and it is thought to add sensitivity and specificity compared with planar bone scans. The addition of SPECT images to bone scans might be helpful in showing a stress fracture of the femur, although no studies have specifically addressed this possibility. 
Stress fractures of the femoral neck can be classified according to their location, which has some prognostic importance. ${ }^{13}$ Superior cortex fractures are thought to be tension-type injuries and have a high propensity to nonunion and development of complete fracture, with possible avascular necrosis. Most surgeons advocate immediate surgical pinning as the treatment of choice. Inferomedial cortex stress fractures are considered compressiontype injuries, and although they have a high complication rate, they do not have as grave a prognosis as tension-sided fractures. These inferomedial side stress fractures can often be treated nonoperatively, usually with prolonged nonweightbearing. ${ }^{13}$

We cared for our patient as if he had a compression-sided stress fracture, even though he had negative findings on a bone scan, equivocal findings on SPECT images, and only mild bone marrow edema on MRI. We did not place him on crutches, however. He had no symptoms when walking, and we did not want to subject him to the inconvenience of crutches and nonweight-bearing status without stronger radiographic evidence of an actual stress fracture. In this case, the patient recovered completely.

Only a few reports exist for stress fractures with negative findings on bone scans. Milgrom et $\mathrm{al}^{14}$ reported three cases of tibial stress fractures in male military recruits who had negative findings on bone scans. Symptoms persisted in all three, and subsequent repeated bone scans were positive. Keene and Lash ${ }^{15}$ described a 17 -year-old female crosscountry runner who had initial negative bone scan findings, but persistent symptoms, and a subsequent MRI showed the femoral neck stress fracture. This case was similar to ours, except that their MRI showed a greater degree of bone marrow edema, and a fracture line was visible. Sterling et $\mathrm{al}^{16}$ described a 42-year-old female marathon runner whose findings on an initial bone scan were negative, but a subsequent plain film was positive for femoral neck stress fracture. Bal and Sandow ${ }^{17}$ described an 81-year-old woman with osteoporosis who developed bilateral femoral neck stress fractures occurring at different times, each with a negative findings on the initial bone scan. They suggest that a bone scan might not be completely reliable in persons with osteoporosis.

In conclusion, we report a case of negative bone scan findings in a 44-year-old man who had MRI evidence of femoral neck bone injury. Whether this injury represented a stress fracture, as usually seen clinically, is unclear. We believe that some sort of bone stress had occurred and was likely the cause of his symptoms. Even so, radionucleotide bone scanning should be considered reliable and sensitive (although maybe not 100\%) for the detection of bone stress injury. False-negative bone scan findings are mainly confined to case reports. Given negative findings on a bone scan, if clinical evidence still strongly suggests stress fracture, either an MRI scan, possibly the addition of SPECT scan, or repeated bone scan should be considered.

\section{References}

1. Bennell KL, Brukner PD. Epidemiology and site specificity of stress fractures. Clin Sports Med 1997; 16:179-96.

2. Matheson GO, Clement DB, McKenzie DC, Taulton JE, Lloyd-Smith DR, MacIntyre JG. Stress fractures in athletes. A case study of 320 cases. Am J Sports Med 1987;15:46-58.

3. Zwas ST, Elkanovitch R, Frank G. Interpretation and classification of bone scintigraphic findings in stress fractures. J Nucl Med 1987;28:452-7.

4. Prather JL, Nusynowitz ML, Snowdy HA. Scintigraphic findings in stress fractures. J Bone Joint Surg Am 1977;59A:869-74.

5. Ishibashi Y, Okamura Y, Otsuka H, Nishizawa K, Sasaki T, Toh S. Comparison of scintigraphy and magnetic resonance imaging for stress injuries of bone. Clin J Sport Med 2002;12:79-84.

6. Roub LW, Gumerman LW, Hanley EN, Clark MW, Goodman M, Herbert DL. Bone stress: a radionuclide imaging perspective. Radiology 1979;132: 431-8.

7. Lee JK, Yao L. Stress fractures: MR imaging. Radiology 1988;169:217-20.

8. Arendt EA, Griffiths HJ. The use of MR imaging in the assessment and clinical management of stress reactions of bone in high-performance athletes. Clin Sports Med 1997;16:291-306.

9. Fredericson M, Bergman AG, Hoffman KL, Dillingham MS. Tibial stress reaction in runners: correlation of clinical symptoms and scintigraphy with a new magnetic resonance imaging grading system. Am J Sports Med 1995;23:472-81.

10. Shin AY, Morin WD, Gorman JD, Jones SB, Lapinsky AS. The superiority of magnetic resonance imaging in differentiating the cause of hip pain in endurance athletes. Am J Sports Med 1996;24:16876.

11. Lusins JO, Elting JJ, Cicoria AD, Goldsmith SJ. SPECT evaluation of lumbar spondylolysis and spondylolisthesis. Spine 1994;19:608-12. 
12. Anderson K, Sarwark JF, Conway JJ, Logue ES, Schafer MF. Quantitative assessment with SPECT imaging of stress injuries of the pars interarticularis and response to bracing. J Pediatr Orthop 2000;20: 28-33.

13. Boden BP, Speer KP. Femoral stress fractures. Clin Sports Med 1997;16:307-17.

14. Milgrom C, Chisin R, Giladi M, et al. Negative bone scans in impending tibial stress fractures. A report of three cases. Am J Sports Med 1984;12:488-91.
15. Keene JS, Lash EG. Negative bone scan in a femoral neck stress fracture. A case report. Am J Sports Med 1992;20:234-6.

16. Sterling JC, Webb Jr. RF Jr, Meyers MC, Calvo RD. False negative bone scan in a female runner. Med Sci Sports Exerc 1993;25:179-85.

17. Bal BS, Sandow T. Bilateral femoral neck fractures with negative bone scans. Orthopedics 1996;19: 974-6. 